\title{
Development and validation of a CGH microarray for clinical cytogenetic diagnosis
}

Sau W. Cheung, $P h D^{1}$, Chad A Shaw, $P h D^{1}$, Wei Yu, $P h D^{2}$, Jiangzham Li, MD, $P h D^{1}$, Zhishuo Ou, $M D^{1}$, Ankita Patel, PhD ${ }^{1}$, Svetlana A. Yatsenko, $M D^{1}$, Mitchell L. Cooper, $B S^{1}$, Patti Furman, $M S^{1}$, Pawal Stankiewicz, MD, $P h D^{1}$, James R. Lupski, $M D, P h D^{1}$, A. Craig Chinault, PhD ${ }^{1}$, and Arthur L. Beaudet, $M D^{1}$

\begin{abstract}
Purpose: We developed a microarray for clinical diagnosis of chromosomal disorders using large insert genomic DNA clones as targets for comparative genomic hybridization (CGH). Methods: The array contains 362 FISH-verified clones that span genomic regions implicated in over 40 known human genomic disorders and representative subtelomeric clones for each of the 41 clinically relevant human chromosome telomeres. Three or four clones from almost all deletion or duplication genomic regions and three or more clones for each subtelomeric region were included. We tested chromosome microarray analysis (CMA) in a masked fashion by examining genomic DNA from 25 patients who were previously ascertained in a genetic clinic and studied by conventional cytogenetics. A novel software package implemented in the R statistical programming language was developed for normalization, visualization, and inference. Results: The CMA results were entirely consistent with previous cytogenetic and FISH findings. For clone by clone analysis, the sensitivity was estimated to be $96.7 \%$ and the specificity was $99.1 \%$. Major advantages of this selected human genome array include the following: interrogation of clinically relevant genomic regions, the ability to test for a wide range of duplication and deletion syndromes in a single analysis, the ability to detect duplications that would likely be undetected by metaphase FISH, and ease of confirmation of suspected genomic changes by conventional FISH testing currently available in the cytogenetics laboratory. Conclusion: The array is an attractive alternative to telomere FISH and locus-specific FISH, but it does not include uniform coverage across the arms of each chromosome and is not intended to substitute for a standard karyotype. Limitations of CMA include the inability to detect both balanced chromosome changes and low levels of mosaicism.
\end{abstract}

Genet Med 2005:7(6):422-432.

Key Words: microarray, FISH, telomere, deletion syndrome, comparative genomic hybridization

Chromosomal imbalances are often associated with multiple congenital anomalies, dysmorphic features, growth retardation, and/or learning disabilities. Many genomic changes causing abnormal phenotypes can be detected by standard cytogenetic methods. Examples of imbalances detected by karyotype include common trisomies and some apparent terminal deletions, such as those associated with Cri-du-chat and WolfHirschhorn syndromes. However, a standard karyotype will not reliably detect deletions or duplications smaller than $4 \mathrm{Mb}$.

Advances in molecular cytogenetics such as the development of locus-specific fluorescence in situ hybridization (FISH) have significantly improved the sensitivity of cytogenetic analysis enabling higher resolution genome analysis. ${ }^{1,2}$

\footnotetext{
From the ${ }^{I}$ Department of Molecular and Human Genetics, Baylor College of Medicine, Houston, Texas; ${ }^{2}$ Codon BioSciences, Houston, Texas.

Sau W. Cheung, PhD, Department of Molecular and Human Genetics, Baylor College of Medicine, One Baylor Plaza, NAB 2015, Houston, TX 77030.

Supplementary data is available online at http://www.geneticsinmedicine.org

Received: February 21, 2005

Accepted: May 5, 2005.
}

DOI: 10.1097/01.GIM.0000170992.63691.32
For example, recurrent microdeletion or duplication events associated with disorders such as Williams-Beuren syndrome ${ }^{3}$ or Charcot-Marie-Tooth disease type 1A (CMT1A) ${ }^{4}$ are readily detected by metaphase or interphase FISH, respectively. The more recent development of FISH probes for subtelomeric regions and simultaneous interrogations of all 41 clinically relevant telomeres has led to the identification of cryptic unbalanced rearrangements in 3\% to $10 \%$ of patients with learning disabilities and mental retardation of otherwise unknown cause. ${ }^{5,6}$ However, telomere FISH is time consuming and labor intensive and provides no information about the extent of the aberration, because only one probe is used for each subtelomeric region.

Comparative genomic hybridization $(\mathrm{CGH})$ was originally applied to metaphase chromosomes for analysis of chromosomal imbalances. ${ }^{7}$ The metaphase chromosomes were later replaced with BAC or PAC (Bacterial or P1 artificial chromosome) clones for hybridization targets immobilized on glass slides as arrays. This enables detection of copy number changes throughout the genome with high resolution and provides a basis for higher throughput analysis of genomic imbalances. ${ }^{8,9}$ Array CGH has been applied for high resolution analysis of 
constitutional abnormalities in specific chromosomal regions, ${ }^{10-12}$ including subtelomeric regions of all chromosomes, ${ }^{13}$ specific targeted chromosome regions such as $15 \mathrm{q} 11.2,{ }^{14}$ and entire chromosomes, such as chromosomes 22 and X. ${ }^{15,16}$ DNA arrays consisting of 2,000 to 4,000 BAC clones representing the sequenced genome at approximately $1-\mathrm{Mb}$ intervals have also been developed. ${ }^{17,18}$ A study using a tiling path DNA microarray with complete coverage of the human genome with 32,433 overlapping BAC clones with the potential to identify disease genes has been reported. ${ }^{19}$ Recently, the use of a genome-wide array has led to the identification of a major gene for the CHARGE syndrome. ${ }^{20}$ However, although extensive coverage of the genome on an array enables higher resolution analysis, this increased resolution is tempered by cost associated with array production, by difficulties in clinical interpretation of newly discovered rare variants, and by current limitations of the knowledge and clinical relevance of large segmental copy number variants. The FDA has issued draft guidance regarding multiplex genetic tests (Federal Register April 21, 2003, Docket No. 03D-0120; http://www.fda.gov/ cdrh/oivd/guidance/1210.html), but it is unclear at present whether tests as complex as described here will be acceptable for FDA approval.

Although most array CGH methods attach unmodified DNA to chemically treated glass, we chemically modify DNA and attach it efficiently to an unmodified glass surface to produce arrays. ${ }^{21}$ Thus, the array of spot has uniform size and shape, critical for precise quantitation. The reliability of this method for detecting small deletions, duplications, or triplications has been well-documented for the chromosome $1 \mathrm{p} 36$ region $^{22}$ and for the proximal $17 \mathrm{p}$ region $^{23}$; in the latter case, four genomic disorders can be distinguished despite a complex organization related to low copy repeats. The focus of the current study was to validate CMA as a routine method for simultaneously screening for DNA copy number changes in clinically relevant regions of the genome, including chromosomal regions associated with more than 40 well-established genomic disorders and 41 subtelomeric regions. ${ }^{24}$ The intent was also to minimize the detection of copy number changes of unknown clinical significance, and focus on well-defined disorders that can be confirmed by conventional FISH tests currently available in the clinical cytogenetics laboratory. To validate this approach, we performed CMA on 25 patients whose diagnoses were previously established in our cytogenetics laboratory.

\section{MATERIALS AND METHODS}

\section{Patient specimens}

Samples from 25 patients with various chromosome abnormalities previously identified by our cytogenetics laboratory were selected for validation studies. All laboratory studies were performed in a masked fashion. Patient samples included 22 from peripheral blood, 2 amniotic fluid cultures, and 1 skin fibroblast culture. The reference DNA samples were derived from peripheral blood of a individual, phenotypically normal male, and female controls with no detectable chromosomal aberrations by conventional karyotype analysis.

\section{Microarray design}

The CMA array was composed of 3 to $10 \mathrm{BAC} / \mathrm{PAC}$ clones per disease locus or subtelomeric region (Table 1), with the exception of three loci; only two clones were included for the subtelomeric region of chromosome $19 \mathrm{q}$, and there was only one clone for the adrenal hypoplasia congenita $(A H C)$ locus and one clone for the $\mathrm{Xq} / \mathrm{Yq}$ telomeric region. A total of 381 clones (287 BAC clones, 83 PAC clones, and 11 cosmids) were selected from NCBI/UCSC public databases or from the FISH probe panel routinely used in the cytogenetic laboratory. The 30 clones covering subtelomeric regions used in the clinical cytogenetics laboratory were obtained from Dr. Jonathan Flint. ${ }^{25}$ Other human genomic clones were from the Roswell Park Cancer Institute (RPCI) BAC and PAC libraries. All clones were FISH-verified before use on the microarray. Any clone that hybridized to multiple chromosomal locations, likely reflecting paralogous sequences, was excluded from the array. In early studies, we identified 19 clones that were polymorphic in the control population or consistently performed poorly on the array, and these were excluded from the final analysis. After removing these clones, our array included 362 distinct clones including 175 clones covering the 41 clinically relevant subtelomeric regions and 187 clones from genomic positions corresponding to more than 40 different genomic disorders (specific information is in the supplementary data available online at http://www.geneticsinmedicine.org).

BAC clone DNA was prepared using standard alkaline lysis methods and chemically modified for array printing as described. ${ }^{22}$ Briefly, the DNA was chemically cross-linked using (3-glycidoxypropyl) trimethoxysilane (Sigma, St. Louis, MO) and printed onto alkaline/acid cleaned Bis(trichlorosily1)octane (Gelest Inc., Morrisville, PA)-treated glass slides (VWR Scientific micro slides cat. no. 48300-047; www.vwr.com) using a Cartesian Technology Axsys printer Model PS5500 (Genomic Solutions, Ann Arbor, MI). All clones were printed in duplicate from a single 384-well plate in spatially distinct subarrays.

\section{DNA labeling and hybridization}

DNA labeling and hybridization were performed as described.22 Genomic DNA was isolated from peripheral blood lymphocytes or cultured amniocytes or fibroblasts using a PureGene DNA-purification kit (Gentra, Systems, Minneapolis, MN), digested with the restriction endonuclease $D p n I \mathrm{I}$ (New England Biolabs, Beverly, MA), and purified by phenol/ chloroform extraction.

Genomic DNAs from patient samples and controls were differentially labeled with cyanin-3 (Cy3) and Cy5 (Perkin Elmer, Boston, MA) using a Bioprime DNA direct labeling kit (Invitrogen, Carlsbad, CA) and hybridized onto the arrays at $37^{\circ} \mathrm{C}$ for 24 hours. The slides were washed and fluorescent signals on the slides were scanned into image files using an Axon microarray scanner and ScanArray software (GenePix 4000B from 
Table 1

Genomic disorders detected by chromosomal microarray analysis

\begin{tabular}{|c|c|c|}
\hline OMIM\# & Disorder & Cytogenetics \\
\hline & 1p36 deletion and/or duplication & 1p36 deletion and/or duplication \\
\hline 194190 & Wolf-Hirschhorn syndrome & $4 \mathrm{p} 16.3$ deletion \\
\hline 123450 & Cri-du-chat syndrome & $5 p 15.2$ deletion \\
\hline 117550 & Sotos syndrome & $5 q 35$ deletion \\
\hline 101400 & Saethre-Chotzen syndrome & $7 \mathrm{p} 21.1$ deletion \\
\hline 175700 & Greig cephalopolysyndactyly syndrome & $7 \mathrm{p} 13$ deletion \\
\hline 194050 & Williams-Beuren syndrome & $7 \mathrm{q} 11.2$ deletion \\
\hline 150230 & Langer-Giedion syndrome & $8 \mathrm{q} 24.11-\mathrm{q} 24.13$ deletion \\
\hline 190350 & Trichorhinophalangeal syndrome, type I & $8 \mathrm{q} 24.12$ deletion \\
\hline 146255 & Hypoparathyroidism, sensorineural deafness \& renal dysplasia & 10p14 deletion \\
\hline 601362 & DiGeorge syndrome 2 & 10p13-p14 deletion \\
\hline 608071 & Split hand/split foot syndrome 3 & 10q24 duplication \\
\hline 130650 & Beckwith-Wiedemann syndrome & 11p15.5 duplication \\
\hline 194072 & Wilm's tumor-aniridia-genitourinary abnormalities syndrome (WAGR) & 11 p13 deletion \\
\hline 601224 & Potocki-Shaffer syndrome & 11p11.2 deletion \\
\hline 180200 & Retinoblastoma & $13 \mathrm{q} 14$ deletion \\
\hline 176270 & Prader Willi syndrome & 15q11.2q13 deletion (paternal) \\
\hline 105830 & Angelman syndrome & 15q11-q13 deletion (maternal) \\
\hline 209850 & Autism & 15q11.2q13 duplication (maternal) \\
\hline 191092 & Tuberous sclerosis 2 & 16 p13.3 deletion \\
\hline 180849 & Rubinstein-Taybi syndrome & 16 p 13.3 deletion \\
\hline 600273 & Polycystic kidney disease/tuberous sclerosis 2 & 16p13.3 deletion \\
\hline 247200 & Miller-Dieker lissencephaly syndrome & 17p13.3 deletion \\
\hline 118220 & Charcot-Marie-Tooth disease type $1 \mathrm{~A}$ & 17p12 duplication \\
\hline 162500 & Hereditary neuropathy with liability to pressure palsies & 17 p12 deletion \\
\hline \multirow[t]{2}{*}{182290} & Smith-Magenis syndrome & 17p11.2 deletion \\
\hline & $\operatorname{dup}(17)(\mathrm{p} 11.2 \mathrm{p} 11.2)$ syndrome & 17p11.2 duplication \\
\hline 162200 & Neurofibromatosis 1 & 17q11.2 deletion \\
\hline 118450 & Alagille syndrome & 20p12 deletion \\
\hline 190685 & Down syndrome & 21q22 duplication \\
\hline 115470 & Cat eye syndrome & $\operatorname{inv} \operatorname{dup}(22)(\mathrm{q} 11.2)$ \\
\hline \multirow[t]{2}{*}{192430,188400} & Velocardiofacial syndrome, DiGeorge syndrome 1 & 22q11.2 deletion \\
\hline & $\operatorname{dup}(22)(\mathrm{q} 11.2 \mathrm{q} 11.2)$ syndrome & 22q11.2 duplication \\
\hline 308100 & Steroid sulfatase deficiency & Xp22.32 deletion \\
\hline 308700 & Kallmann syndrome 1 & Xp22.3 deletion \\
\hline 309801 & Microphthalmia with linear skin defects & Xp22.31 deletion \\
\hline 300200 & Adrenal hypoplasia, congenital & Xp21.2-21.3 deletion \\
\hline 300474 & Glycerol kinase deficiency & Xp22 deletion \\
\hline 312080 & Pelizaeus-Merzbacher disease & Xq22 duplication or deletion \\
\hline 480000 & Testis-determining factor on $\mathrm{Y}$ & Yp11.2 deletion \\
\hline \multirow[t]{3}{*}{415000} & Azospermia (factor a) & Yq11 deletion \\
\hline & Azospermia (factor b) & Yq11 deletion \\
\hline & Azospermia (factor c) & Yq11 deletion \\
\hline
\end{tabular}


Axon Instruments, Union City, CA). For each patient sample, two experiments were performed with reversal of the dye labels for the control and test samples, and the data from both dyereversed hybridizations were integrated to determine inferences for each case.

Gender-reversed controls that served as initial positive controls in our study were used in six cases; gender-matched controls were used in the remaining 19 cases. Because the number of clones on the sex chromosomes is large (73/362), we excluded the sex chromosomes clones for these 6 cases from our power and sensitivity calculations. Thus, the full data set considered for our power and specificity analyses consisted of 8612 $(362 \times 19)+(289 \times 6)$ data points with 4 replicates (i.e., two from each hybridization).

\section{Data processing}

Microarray image files were quantified using GenePix Pro 5 software. The quantitation data were subjected to normalization as described previously, ${ }^{23}$ and the dye-reversed data were combined to determine a single fold-change value for each clone. Inferences were made for all clones using these final combined data values. All analyses were performed on $\log _{2}$ ratios using code for the normalization and inference that was implemented in the $\mathrm{R}$ statistical programming language.

Justification for data normalization in spotted arrays is well documented. Our normalization procedure removes systematic biases such as spatial and intensity artifacts within arrays as well as shifts and scale changes between arrays. Briefly, the steps are (1) spot rejection based on robust estimation (L2e) of a Gaussian distribution for spot backgrounds and spot intensitites followed by identification of those spots more than 3 standard deviations from the mean, ${ }^{26}$ (2) quantile normalization to balance single channel intensities of the accepted spots for each array, ${ }^{27}$ (3) spatial normalization of log-ratio values within individual arrays using a loess (a nonparametric smoothing function) regression procedure with a smoothing parameter of 0.35 , (4) averaging of normalized within array replicate spots, (5) bi-chip scaling between the pair of arrays to remove scale changes between the dye-reversed pair $^{28}$ followed by a shift adjustment to ensure that each array has a mean 0 log-ratio value, and finally, (6) sign-change of one array and averaging of the data for each clone between the dye-reversed pair of arrays. ${ }^{23}$

The normalization results in a single combined data value and variance estimate for each clone and for each patient. These combined data are used to make inferences regarding the gain/loss/no change status of regions detected by each clone for each patient. In this article, we present an exceedingly simple inference method. We select uniform cutoff of 0.2 for the Test/Reference ratios, and we call clones "gain" when the dye-reversed combined data exceeds the 0.2 magnitude. We use a symmetric cutoff of -0.2 on the combined data to call clones "loss."

\section{FISH analysis}

Metaphase spreads were prepared from patient derived lymphocytes using standard procedures. Miniprep DNA (500 ng) was labeled with biotin-14-dUTP or digoxigenin-11-dUTP by standard nick translation, and 100 ng of labeled DNA was used for FISH analysis using standard protocols. ${ }^{29}$

\section{Validation procedure}

The stepwise evaluation process for a clone showing a gain or loss is first to review an interactive database displaying profile consisting of "raw," "normalized," and "combined" data. Initially, FISH confirmation was performed in situation wherever the T/R ratios exceed or below the 0.2 cutoff. When in situations where FISH analysis failed to confirm the gain or loss, we classified these clones as false positive clones.

\section{RESULTS}

\section{Validation of chromosomal abnormalities and microdeletions}

Chromosome microarray analysis was used in a masked fashion to examine 25 samples that were selected from the Baylor cytogenetics laboratory representing various chromosomal abnormalities and microdeletions. CMA results were compared to G-banding analysis in 10 cases (Table 2) and to FISH analysis of microdeletion syndromes in 15 cases (Table 3). In each case, CMA correctly detected the previously identified abnormalities, and in three cases, the microarray identified additional cryptic microdeletions and/or microduplications that were subsequently verified by FISH analysis.

An example of CMA on a representative patient (case $\mathrm{K} 4$ ) is shown in Figure 1. Case K4 was a phenotypic male with a karyotype of 46,X,der(X)t(X;Y)(p22.33;p11.2) [partial karyotype shown in Figure 1B] that was verified by FISH analysis using the SRY probe as shown in Figure 1D. The actual array and subarray using control female DNA are shown in Figure 1A. Figure $1 \mathrm{C}$ shows the microarray profile of the raw data (left), i.e., the test/reference ratios (T/R ratio) of two experiments with dye swap; normalized data (middle); and the combined data (right) with sign change of one array. In this experiment, a copy number gain was detected by four clones at the Yp11.3 region as expected, with a T/R ratio ranging from 0.46 to 0.569 compared to the selected gain threshold of 0.20 . The array profile displayed the four clones spanning approximately $2.5 \mathrm{Mb}$ of genomic sequence on Yp11.3 as a region of copy number gain, thus defining this genomic interval more precisely than the standard cytogenetic and FISH methods.

Another example of microarray results is depicted in Figure 2, which represents a DNA sample derived from cultured amniocytes (identified as K10 in Table 2). The microarray result for this case indicate a genomic loss detected by 10 target clones in the array corresponding to the 4 p16.3 region, reflecting a terminal deletion of chromosome $4 \mathrm{p}$. The array profile displayed all 10 clones with $T / R$ values ranging from -0.378 to -0.658 , well below the -0.2 cut off chosen for copy number loss. Thus, the microarray finding is completely consistent 
Table 2

Comparison of karyotype abnormalities to the copy number changes detected by CMA

\begin{tabular}{|c|c|c|c|c|c|c|}
\hline \multirow[b]{2}{*}{ No. } & \multirow[b]{2}{*}{ Indication } & \multirow[b]{2}{*}{ Karyotype } & \multicolumn{3}{|c|}{ Chromosomal microarray analysis } & \multirow[b]{2}{*}{ Comparison } \\
\hline & & & $\begin{array}{l}\text { No. clones } \\
\text { in region }\end{array}$ & $\begin{array}{c}\text { No. clones } \\
\text { with gain/loss }\end{array}$ & $\begin{array}{l}\text { No. clones } \\
\text { no gain/loss }\end{array}$ & \\
\hline K1 & Rule out trisomy 21 & $46, \mathrm{XX}, \operatorname{der}(21 ; 21)(\mathrm{q} 10 ; \mathrm{q} 10),+21$ & 7 & 7 & 0 & Consistent \\
\hline K2 & Possible Angelman syndrome & 46,XY,del(13)(q33.2) & 4 & 3 & $1^{a}$ & Additional findings \\
\hline K3 & Developmental delay & 46,XX,inv dup (8)(p11.23p23.1) & 5 & 5 & 0 & Additional findings \\
\hline K4 & Rule out Klinefelter syndrome & 46,X,der $(\mathrm{X}) \mathrm{t}(\mathrm{X} ; \mathrm{Y})(\mathrm{p} 22.33 ; \mathrm{p} 11.3)[\mathrm{SRY}+]$ & 4 & 4 & 0 & Consistent \\
\hline K5 & Dysmorphic; psychiatric disorder & $46, \mathrm{XX}, \operatorname{del}(22)(\mathrm{q} 11.21 \mathrm{q} 11.23)$ & 4 & 4 & 0 & Consistent \\
\hline K6 & Rule out trisomy $13 / 18$ & $47, \mathrm{XY},+18$ & 14 & 13 & $1^{b}$ & Consistent \\
\hline K7 & Developmental delay; dysmorphic & $47, \mathrm{XXY}$ & 54 & 50 & 4 & Consistent \\
\hline K8 & Langer-Giedion syndrome & $46, \mathrm{XX}, \operatorname{del}(8)(\mathrm{q} 24.1 \mathrm{q} 24.1)$ & 7 & 7 & 0 & Consistent \\
\hline K9 & Multiple congenital anomalies & 46,XX,del(18)(p11.2) & 6 & 6 & 0 & Consistent \\
\hline \multirow[t]{2}{*}{ K10 } & Abnormal fetal ultrasound & $46, \mathrm{XX}, \operatorname{del}(4)(\mathrm{p} 14)$ & 10 & 10 & 0 & Consistent \\
\hline & Total & & 115 & 109 & 6 & \\
\hline
\end{tabular}

${ }^{a}$ Clone $\mathrm{T} / \mathrm{R}$ ratio -0.185 [Loss].

${ }^{b}$ Clone T/R ratio -0.184 [Loss].

Table 3

Comparison of microdeletions/duplications to the copy number changes detected by CMA

\begin{tabular}{|c|c|c|c|c|c|c|}
\hline \multirow[b]{2}{*}{ No. } & \multirow[b]{2}{*}{ Indication } & \multirow[b]{2}{*}{ FISH analysis } & \multicolumn{3}{|c|}{ Chromosomal microarray analysis } & \multirow[b]{2}{*}{ Comparison } \\
\hline & & & $\begin{array}{l}\text { No. } \\
\text { clones in } \\
\text { region }\end{array}$ & $\begin{array}{l}\text { No. clones } \\
\text { with } \\
\text { gain/loss }\end{array}$ & $\begin{array}{l}\text { No. clones } \\
\text { no gain/loss }\end{array}$ & \\
\hline $\mathrm{F} 1$ & Intellectual impairment; minor congenital abn. & ish del(1)(q44)(D1S3738-) & 8 & 8 & 0 & Consistent \\
\hline $\mathrm{F} 2$ & Bilateral Wilms tumor; hypospadias & ish del(11)(p13p13)(D11S324-) & 2 & 2 & 0 & Consistent \\
\hline F3 & Developmental delay; short stature & ish $\operatorname{del}(7)(\mathrm{q} 11.23 \mathrm{q} 11.23)(\mathrm{ELN}-)$ & 8 & 8 & 0 & Consistent \\
\hline $\mathrm{F} 4$ & Dysmorphic features & ish del(8)(q24.1q24.1)(EXT1-,TRPS1-) & 7 & 7 & 0 & Consistent \\
\hline F5 & Possible neurofibromatosis & ish $\operatorname{del}(17)(\mathrm{q} 11.2 \mathrm{q} 11.2)(\mathrm{NF} 1-)$ & 3 & 3 & 0 & Consistent \\
\hline F6 & Possible DiGeorge/velocardiofacial syndrome & ish del (22)(q11.2q11.2)(F5-,D22S75-) & 4 & 4 & 0 & Consistent \\
\hline F7 & Failure to thrive & ish $\operatorname{del}(22)(\mathrm{q} 13.33)(\mathrm{MS607}-)$ & 4 & 4 & 0 & Additional finding \\
\hline F8 & Rule out adrenal hypoplasia & ish $\operatorname{del}(\mathrm{X})(\mathrm{p} 21 \mathrm{p} 21)(\mathrm{DAX} 1-)$ & 4 & 4 & 0 & Consistent \\
\hline F9 & Hereditary neuropathy with liability to pressure & ish del(17)(p12p12)(PMP22-) & 5 & 5 & 0 & Consistent \\
\hline F10 & Possible Charcot-Marie-Tooth disease & nuc ish 17 p12(PMP22×3) & 5 & 5 & 0 & Consistent \\
\hline F11 & Possible Charcot-Marie-Tooth disease & nuc ish 17 p12(PMP22×3) & 5 & 5 & 0 & Consistent \\
\hline F12 & Low estriol on maternal serum screen & 46,XY.ish del(X)(p22.3p22.3)(STS-) & 3 & 3 & 0 & Consistent \\
\hline F13 & Ichthyosis & ish $\operatorname{del}(\mathrm{X})(\mathrm{p} 22.3 \mathrm{p} 22.3)(\mathrm{STS}-)$ & 3 & 3 & 0 & Consistent \\
\hline F14 & Child with Pelizaeus-Merzbacher disease & nuc ish $\mathrm{Xq} 22(\mathrm{PLP} \times 3)$ & 3 & 3 & 0 & Consistent \\
\hline \multirow[t]{2}{*}{ F15 } & Family history of Pelizaeus-Merzbacher disease & nuc ish $\mathrm{Xq} 22(\mathrm{PLP} \times 3)$ & 3 & 3 & 0 & Consistent \\
\hline & Total & & 67 & 67 & 0 & \\
\hline
\end{tabular}

with the $4 \mathrm{p}$ deletion observed in the conventional cytogenetic studies, as shown in Figure 2.

For case F8 in Table 3, a deletion at the adrenal hypoplasia $(A H C)$ locus was detected by conventional FISH analysis, and the array confirmed this finding. Furthermore, the array displayed a loss of three additional clones encompassing the glycerol kinase region (data not shown). All four clones gave $\mathrm{T} / \mathrm{R}$ ratios ranging from -0.52 to -0.86 , again well below the -0.2 cut off for copy number loss, suggesting the presence of a contiguous gene deletion syndrome including the $A H C$ and glycerol kinase loci. 
A.

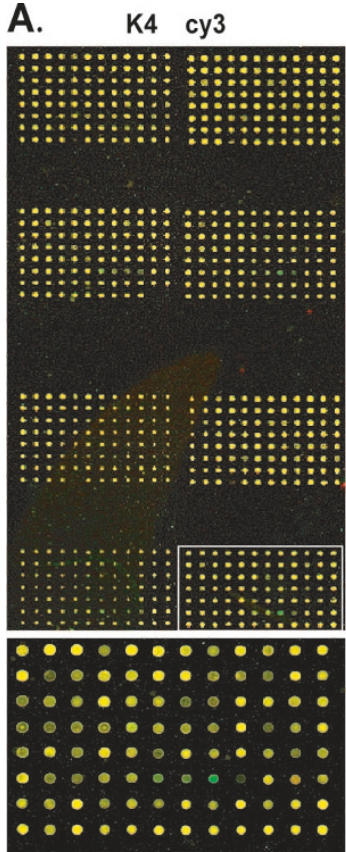

B.

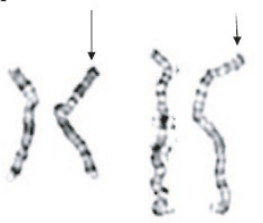

46,X,der $(X) t(X ; Y)(p 22.33 ; p 11.2)$

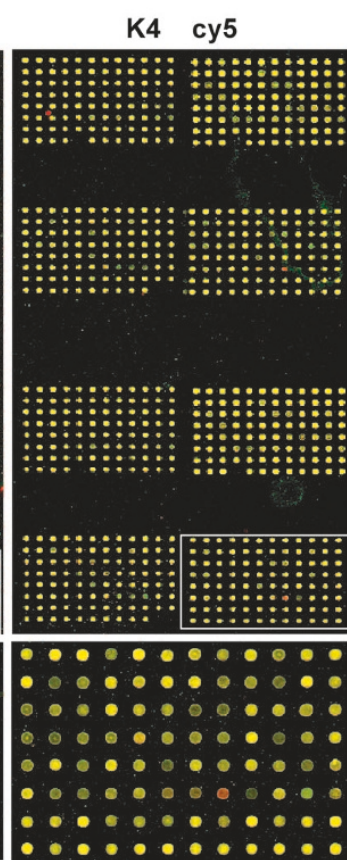

D.

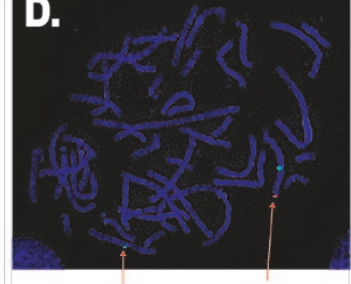

SRY
C. Raw Normalized Combined

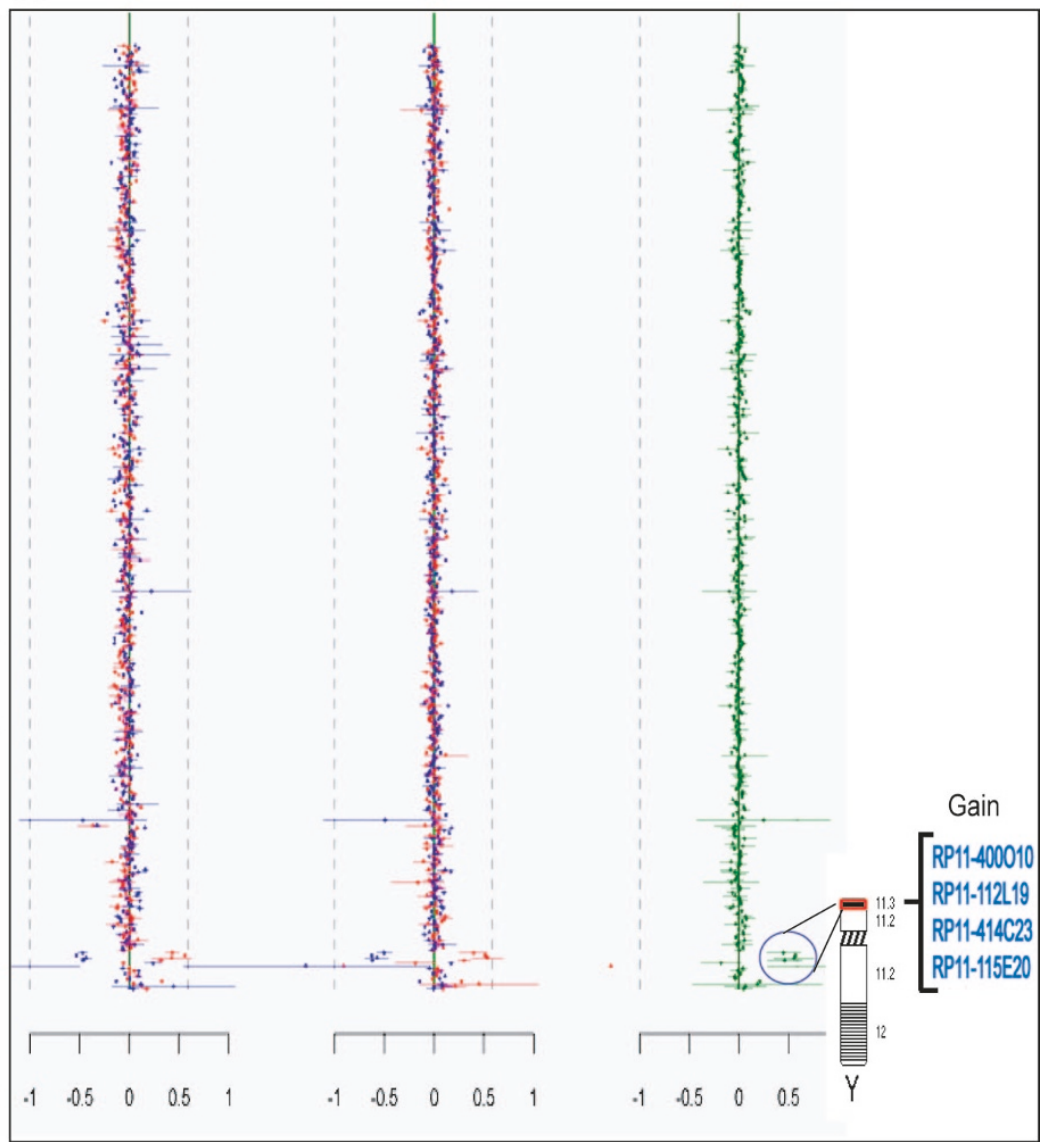

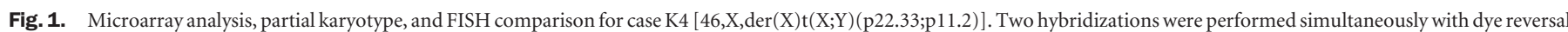

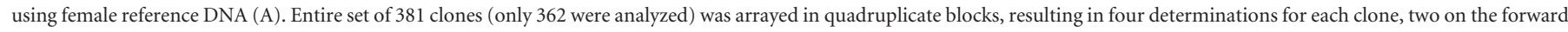

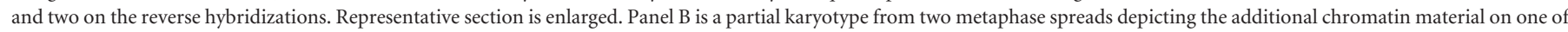

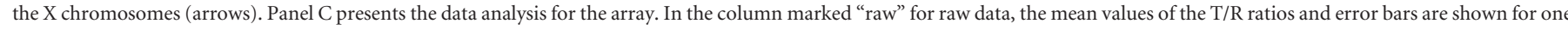

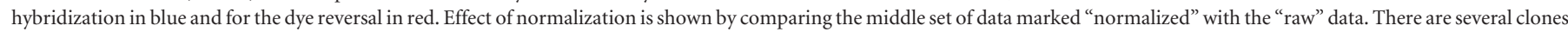

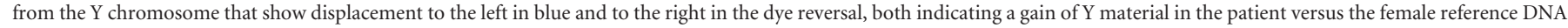

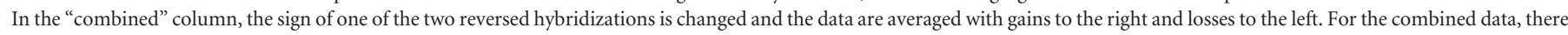

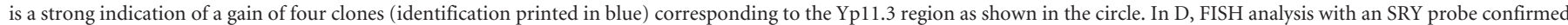
the presence of Y DNA on the short arm of one X chromosome.

\section{Additional abnormalities identified by CGH microarray}

The karyotype of case K2 (Fig. 3) indicated a subtle terminal deletion on the distal long arm of chromosome 13 [46,XY,del(13)(q33.2)], which was subsequently confirmed by FISH analysis with a telomeric probe for chromosome 13 (Figs. 3B and 3C). Microarray analysis (Fig. 3A) revealed deletion of three of the four clones representing the telomere of chromosome $13 \mathrm{q}$ and encompassing approximately $3 \mathrm{Mb}$ of the genomic sequence confirming the presence of a deletion of 13q33.2. In addition, a single clone located in the subtelomeric region of 22q (GS99K24), with an estimated maximum physical distance of 100 to $300 \mathrm{~kb}$ from the telomere, also revealed a copy number loss. This finding was subsequently confirmed by FISH analysis with the same clone present on the microarray, as shown in Figure 3D. Therefore, microarray analysis in this patient not only confirmed the cytogenetic abnormality with more precision but also identified a cryptic deletion that was not identified by routine high-resolution chromosome analy- sis. It is unclear if the $22 \mathrm{q}$ loss is a benign variant or if it contributes to the phenotypic abnormalities in this patient, particularly because CMA could not be performed on the parents. There is precedent for submicroscopic deletions of this chromosome 22 region causing mental retardation and speech delay. ${ }^{30}$

In another case (K3 in Fig. 4), cytogenetic analysis showed an inversion and duplication of the distal short arm of one chromosome 8 [46,XX,inv $\operatorname{dup}(8)(\mathrm{p} 11.23 \mathrm{p} 23.1)]$. The inv $\operatorname{dup}(8)$ was deleted for a subtelomeric FISH probe for $8 \mathrm{p}$ as is commonly observed for this abnormality. ${ }^{31,32}$ The CMA profile revealed a loss of four clones on the subtelomeric region of $8 \mathrm{p}$ with $\mathrm{T} / \mathrm{R}$ values ranging from -0.435 to -0.71 and a gain of one clone (RP11-520F7) at 8p22, confirming the previously reported cytogenetic anomaly. In addition, a gain was found for two clones from the chromosome $18 \mathrm{q}$ telomere (Fig. 4B). This gain was subsequently verified by FISH analysis and was shown to be localized to the short arm of the inv dup(8) (Fig. 


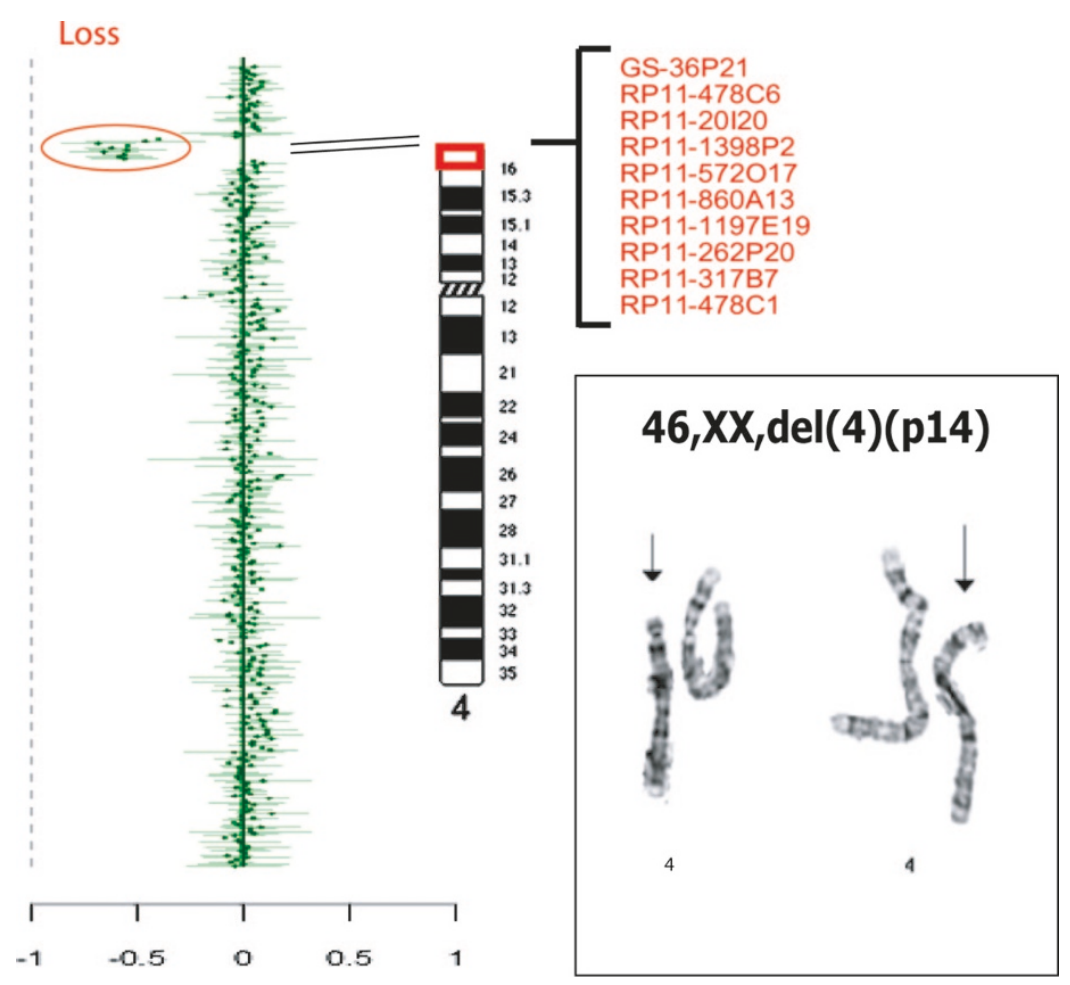

Fig. 2. Microarray analysis and partial karyotype comparison for case $\mathrm{K} 10$ [46,XX,del(4)(p14)]. Microarray profile of the combined data are shown on the left indicating a loss for 10 clones corresponding to the clones in the array for the 4 p 16.3 region. Identification of deleted clones is printed in red. Finding is consistent with the cytogenetic finding of a $4 \mathrm{p}$ deletion from cultured amniocytes as shown on the right.

4C). Therefore, CMA not only confirmed the duplication of the distal short arm of one chromosome 8 and the loss of the $8 p$ telomeric region but also identified a cryptic duplication of the $18 \mathrm{q}$ telomeric region. However, it is also possible, though unlikely, that the gain of two clones from the $18 \mathrm{q}$ telomeric region may represent copy number polymorphism. Because this is conducted in a masked fashion, parental studies are unavailable for the array analysis.

For case F7 (Table 3), subtelomeric FISH analysis identified a deletion on the distal long arm of one chromosome 22 at band q13.3. The microarray profile demonstrated deletion of all four target clones with T/R ratios ranging from -0.535 to -0.651 , confirming and better defining a loss in the 22q13.3 deletion as indicated by the subtelomeric FISH assay. In addition, a single clone located in the distal end of chromosome $14 \mathrm{q}$ (GS820M16), with an estimated maximum physical distance of 100 to $300 \mathrm{~kb}$ from the telomere, revealed a copy number gain with a ratio of 0.252 . This gain was subsequently confirmed by interphase FISH with the same clone present on the microarray. The analysis revealed a sizable deletion encompassing a $130 \mathrm{~kb}$ region at $22 \mathrm{q}$ and a cryptic duplication of one clone at the telomeric region of chromosome 14 that would otherwise be missed by the conventional cytogenetic and /or metaphase FISH methods. Although the parents were not available for study, this duplication is likely a benign variant because segmental duplication of this region is known to occur. ${ }^{33}$

\section{Sensitivity and specificity of individual clones}

The overall single-clone true positive detection rate was 176/ 182 , or $96.7 \%$. In every case $(100 \%)$ where multiple clones were present for a locus, the microarray detected a change in the majority of the clones at the abnormal locus. All six putative false-negative clones where the T/R ratios did not exceed the threshold were from the group with known chromosome abnormalities as shown in Table 2. Four of the 54 clones on the $\mathrm{X}$ chromosome failed to detect the copy number changes in case K7 (Klinefelter syndrome). The T/R ratios for the remaining two cases (one clone per case) were slightly below the 0.2 cutoff, but were detectable deletions by visual inspection. Interestingly, as indicated in Table 3, there was 100\% agreement for 67 clones in 15 cases between the target clones in each of the disease regions and the number of clones detected in cases with known microdeletions. Furthermore, the sensitivity to detect submicroscopic duplications as well as deletions spanning $\approx 1.5 \mathrm{Mb}$ of the genome region was reproducibly validated, as demonstrated in the cases involving hereditary neuropathy with liability to pressure palsies/Charcot-Marie-Tooth disease type 1A (HNPP/CMT1A) region. The same number of target clones in a given region was consistently detected in two cases involving the steroid sulfatase (STS) region, in two cases with changes in the Pelizaeus-Merzbacher disease (PMD) region, in two cases of Langer-Giedion syndrome (LGS), and in two cases of DiGeorge syndrome (DGS). The deletion in the DiGeorge 


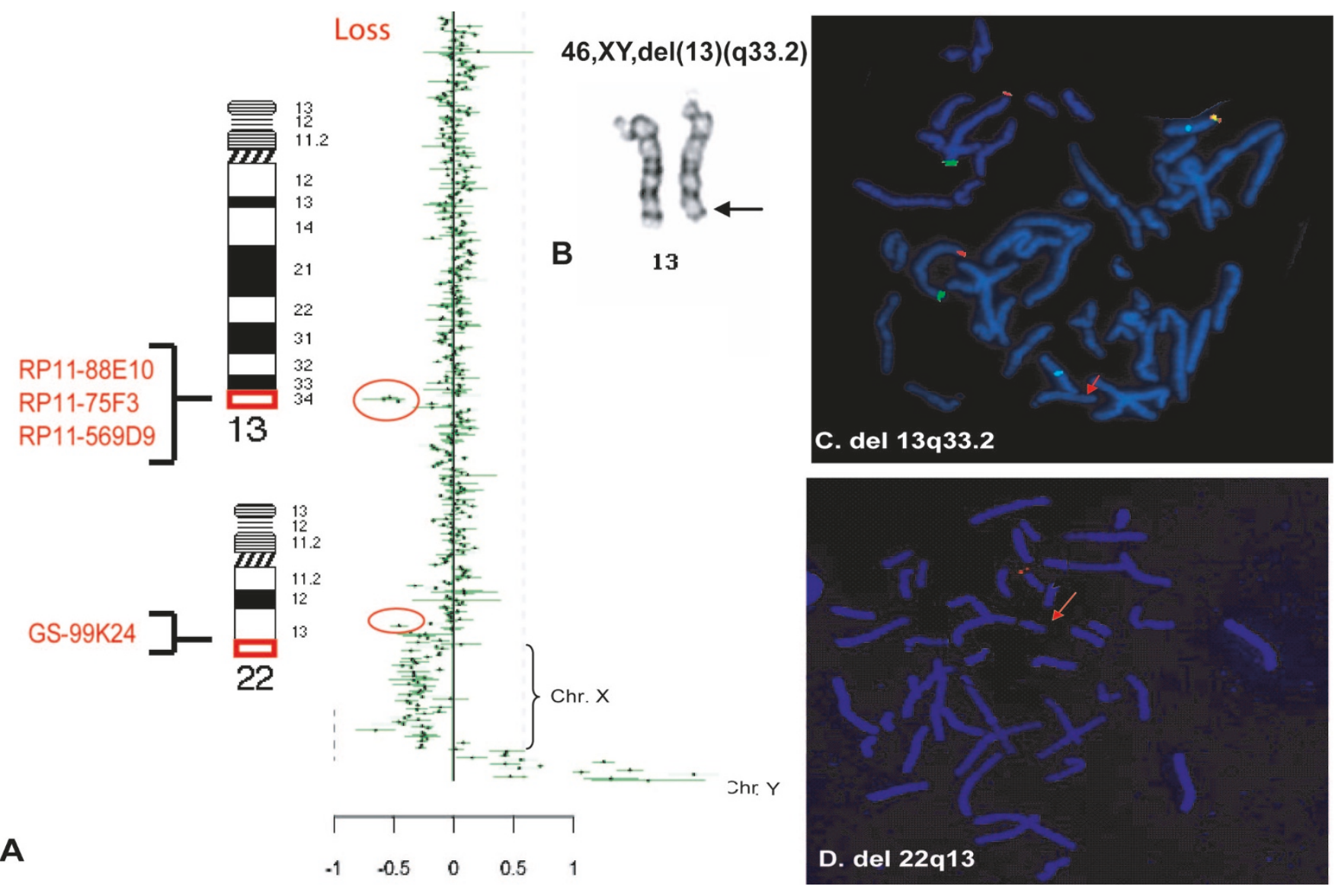

Fig. 3. Microarray analysis, partial karyotype, and FISH comparison for case K2 [46,XY,del(13)(q33.2)]. Microarray data are shown in panel A with gender-reversed reference DNA demonstrating loss for the $\mathrm{X}$ chromosome and gain for the $\mathrm{Y}$ chromosome as expected and also a deletion of four clones in the $13 \mathrm{q} 34$ region. Data are in agreement with the partial karyotype in 3B and FISH analysis in 3C. In addition, the loss of a single clone on the terminal end of chromosome 22 was observed and subsequently was confirmed by FISH analysis as shown in 3D. Identification of deleted clones is printed in red.

syndrome critical region involved the common 3-Mb region in both cases.

Because of the large multiple testing arrangement in microarray settings, array CGH can potentially generate falsepositive results. The empirical cutoff of 0.2 was established to minimize no false negatives, but was designed to be very liberal about the possibility of false positive, which could then be examined by other approaches to decide whether or not they were significant. There were 82 instances of "false-positive" signals defined as a $\mathrm{T} / \mathrm{R}$ ratio exceeding the threshold of \pm 0.2 in a region that was karyotypically normal. This is out of 8612 tests $[(362 \times 19)+(289 \times 6)]$ in 25 cases. For $7 / 82$ false positives, there was major discordance between the two reversed hybridizations, and these likely represented technical artifacts. For one case, FISH confirmed a copy number change that is likely to represent a benign variant, and thus this represented a true positive. None of the remaining 74 signals were associated with a copy number change detected by FISH; these included 52 clones seen in only one patient, eight clones observed in two patients each, and one clone observed in six patients. In unpublished experience in clinical applications, changes of this type usually are found in one normal parent, and these clones may detect lower frequency, short segment benign variants.

\section{DISCUSSION}

The purpose of this study was to develop and validate a CGH microarray that could provide an alternative to telomere FISH and disease-specific FISH in the cytogenetic diagnostic laboratory. The intent was neither to substitute for a routine karyotype nor to achieve dense genomic coverage to discover novel abnormalities currently not detectable in routine FISH studies. This study confirms the utility of array-CGH technology as an alternative to locus-specific FISH and telomere FISH. The CMA array contains multiple genomic clones for each diseasespecific region and for subtelomeric regions of clinical relevance. The microarray is designed to provide redundancy with high sensitivity and specificity for detection of well-characterized disorders, while at the same time minimizing detection of variations of uncertain clinical significance. These CMA results further demonstrate the great potential of arrays for clinical diagnosis. In the study of 25 cases, the overall results from CMA were completely consistent with the known karyotypic and FISH abnormalities. For clone-by-clone analysis, the sensitivity was estimated to be $96.7 \%$ and the specificity was $99.1 \%$. The array also detected some previously undetected imbalances. 

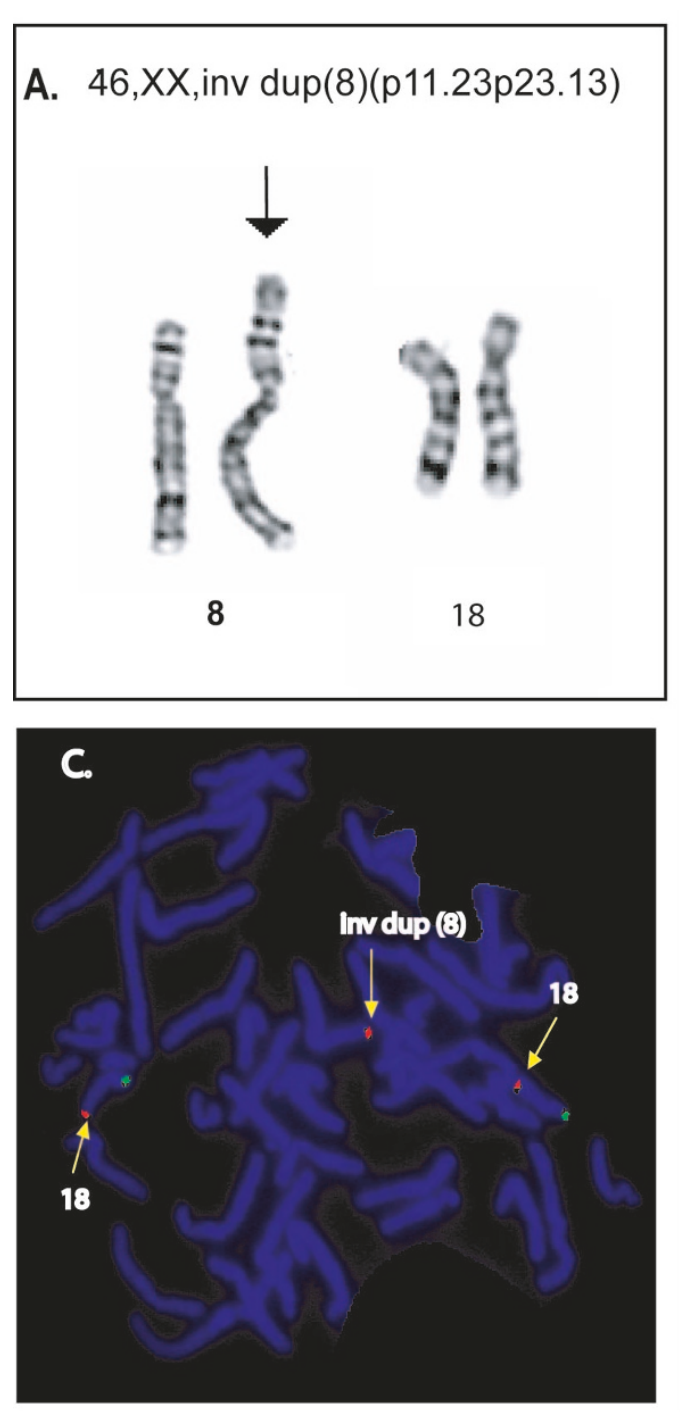

B.

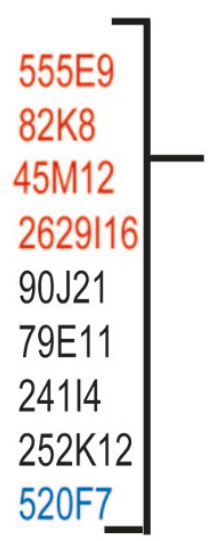

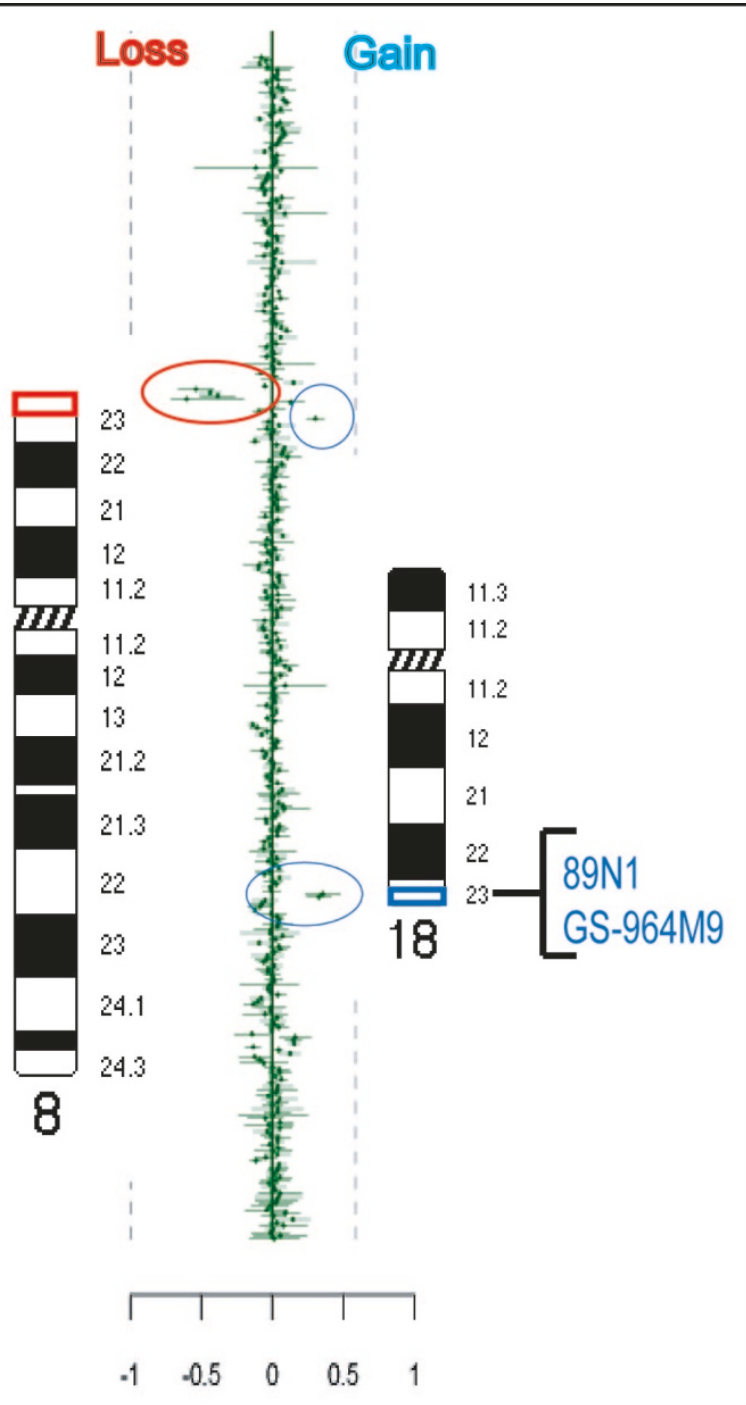

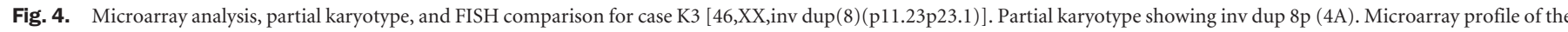

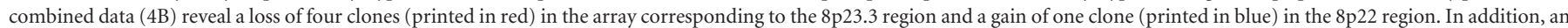

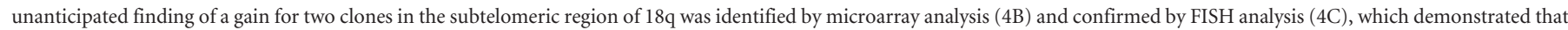
the additional copy of $18 \mathrm{q}$ material was located at the distal short arm of the inv dup chromosome 8 ; red probe is $18 \mathrm{qter}$ and green probe is $18 \mathrm{pter}$.

The choice of whether to use DNA from an individual or from a pool of individuals as the control is an important variable in array CGH. Although use of opposite sex control DNA provides evidence of differing copy number as an internal standard and may be useful for protocol development, this prevents optimal analysis for gain or loss on the X and Y chromosome, and we have opted for same sex control DNA in future clinical studies. We prefer individual control DNA over pooled DNA because the interpretation of what appears to be a polymorphisms may be confused by the averaging of copy number in pooled samples. Because of the variability in frequency of apparent polymorphisms at different regions, in controlled experiments, the use of pools tended to introduce more noise in the system leading to the need for additional follow-up studies to resolve the significance of intermediate results. In contrast, differences between single individuals were much more obvi- ous and have allowed us to collect better data on clones/regions affected by this with the goal of improving the assay.

An attractive option might be the use of control DNA from cytogenetically stable, thoroughly characterized, individual cultured fibroblast cell lines available through repositories. We are exploring the possibility of spiking control or test DNA in various ways to introduce internal standards.

Distinguishing benign genomic variants from disease-causing gains or losses can be challenging even when using clones selected for low rates of polymorphism. In some circumstances, this is similar to the experience with telomere FISH, but there is the added possibility that smaller segments of gain or loss may be detected by array CGH, but not by conventional FISH with large insert clones. An initial important variable for interpretation is whether any FISH or CGH variant is present in a phenotypically normal parent or is a de novo finding in a symptomatic child. 


\section{Comparison of CMA with standard chromosome and FISH analysis}

Array CGH will not detect balanced translocations or inversions, which will remain best detected by karyotype or other methods. In the case of unbalanced translocations, karyotype and/or telomere FISH are required to distinguish a normal parent from a balanced translocation parent. The CMA array is designed to offer an alternative to the use of telomere FISH and selected disease-specific FISH. This array was designed to provide substantial redundancy both by including multiple clones for each clinically relevant region and by duplicate analysis with dye-reversal. Significant advantages of this CMA format are (1) the ability to diagnose atypical phenotypes in cases of well-characterized disorders, (2) the ability to detect duplications that ordinarily require interphase FISH which is rarely used, (3) the ability to diagnose nonspecific phenotypes where it is difficult for the clinician to recognize the need for a specific FISH test, (4) the potential to test postmortem samples and tissues from macerated stillbirths, and (5) the ability to detect the diagnosis independent of the dysmorphology skills of the clinician and independent of the quality of metaphase or prometaphase preparations.

CMA may represent an attractive alternative to repeating a karyotypic analysis. Geneticists frequently repeat chromosome analysis after a previous prenatal or postnatal analysis in order to obtain better prometaphase preparations, and it is moderately common to detect previously unrecognized abnormalities, although this is not well documented in published literature to our knowledge. Most of these abnormalities would be detected by the microarray described in this study. For example, many of the cases of 1 p36 deletion diagnosed in our cytogenetic laboratory had previous chromosome studies reported to be normal. Thus, CMA may be an attractive alternative to repeating a conventional karyotype. CMA can provide the data that would ordinarily be provided by telomere FISH and by dozens of specific FISH tests. In addition, CMA has the ability to define the extent of an imbalance, because multiple clones per telomere and per disease region are utilized (e.g., distinguishing class I and class II deletions in the Prader-Willi/Angelman region). ${ }^{34}$ The major disadvantages of the test at present are not medical but rather administrative and pragmatic and include cost, problems of insurance reimbursement, and limited availability.

CMA identified imbalances that were undetected by karyotype and routine FISH methods. One such example is case K3 in which a deletion of the $8 \mathrm{p}$ subtelomeric region spanning $>7$ $\mathrm{Mb}$ was apparently stabilized through the acquisition of the $\approx 0.5 \mathrm{Mb}$ subtelomeric region of $18 \mathrm{q}$. This cryptic rearrangement probably involved a telomere capture mechanism by nonhomologous recombination mediated by repetitive elements. ${ }^{35-37}$ Another finding undetected by conventional studies but identified by CMA was a deletion of a single clone in the subtelomeric region of one chromosome 22 in case K2. A terminal deletion of $22 \mathrm{q}$ of $130 \mathrm{~kb}$ in size has been associated with severe speech delay and autistic features, ${ }^{38,39}$ but its significance is not clear in this case. In case F8, a $2 \mathrm{Mb}$ deletion of multiple clones spanning from the adrenal hypoplasia congenital (AHC) region to the glycerol kinase (GK) region was detected by CMA, although the initial indication for the FISH study was to rule out a deletion for only the AHC region. Thus, this patient has a contiguous gene deletion syndrome. ${ }^{24} \mathrm{Al}-$ though CMA identified these previously undetected abnormalities, the clinical significance remains to be determined.

\section{Statistical analysis of array data}

The field of genomic microarray analysis using CGH is new relative to that of gene expression array analysis. Fortunately, the same normalization and data processing schemes that have proven successful for expression arrays also perform well for chromosomal CGH arrays. A challenge with any array study is the problem of multiple comparisons. In our studies, we utilized an extremely simple inference method, yet despite its simplicity, the method proved to be both powerful and specific in detecting chromosomal changes. Because the CMA array has many clones representing each genomic locus, the evidence for overlapping or adjacent clones can be considered jointly to determine the inference for each region. We found $100 \%$ concordance among same-locus clones in every case. Furthermore, in instances where our array inference was determined to be a false-positive result (a rate of 0.0095), it mostly occurred as a single inconsistent clone and tended to cluster in various subtelomeric regions. In general, the use of redundant, overlapping clones on the CMA array allows the analyst to identify clones with discordant data values that are likely to represent technical variability rather than true gains or losses.

\section{Genomic polymorphisms}

There is substantial and growing evidence of large segmental polymorphisms in the human genome, ${ }^{18,40-44}$ and there is particular evidence of polymorphisms in telomeric regions ${ }^{45}$ based in part on clinical experience with telomere FISH. ${ }^{6} \mathrm{Be}-$ cause the samples used in this study were not obtained under conditions where parental samples were always available, the data reported here are not optimal for distinguishing benign from disease-causing variants. Despite this limitation, there was suggestive evidence of benign variants in the samples. This included multiple clones as enumerated in Results where the microarray detected a dosage change that was not confirmed by FISH. Some or all of these may represent benign variation in copy number for a portion of the genomic clone.

In summary, array CGH is proving to be an exceedingly accurate method to detect copy number changes in the genome. The collection of 25 patients in our study represented more than 20 distinct genomic disorders, and the array was able to detect the known anomaly in all cases. Confidence in our results is heightened because this array contains multiple genomic clones to assess each region and includes duplicate analysis with dye reversal resulting in excellent concordance between CMA and traditional methods. 


\section{ACKNOWLEDGMENTS}

We thank Jonathan Flint for providing many telomeric clones and the cytogenetic core facility of the Mental Retardation and Developmental Disabilities Research Center (NIH HD 24064) for FISH mapping and end sequencing of clones. The Department of Molecular and Human Genetics at Baylor College of Medicine offers microarray testing on a fee basis. The Department also has a founding relationship with Spectral Genomics, Inc.

\section{References}

1. Langer-Safer PR, Levine M, Ward DC. Immunological method for mapping genes on Drosophila polytene chromosomes. Proc Natl Acad Sci USA 1982;79:4381-4385.

2. Pinkel D, Straume T, Gray JW. Cytogenetic analysis using quantitative, high-sensitivity, fluorescence hybridization. Proc Natl Acad Sci USA 1986;83:2934-2938.

3. Ewart AK, Morris CA, Atkinson D, Jin W, Sternes K, Spallone P et al. Hemizygosity at the elastin locus in a developmental disorder, Williams syndrome. Nat Genet 1993;5:11-16.

4. Lupski JR, de Oca-Luna RM, Slaugenhaupt S, Pentao L, Guzzetta V, Trask BJ et al. DNA duplication associated with Charcot-Marie-Tooth disease type 1A. Cell 1991; 66:219-232.

5. Flint J, Wilkie AOM, Buckle VJ, Winter RM, Holland AJ, McDermid HE. The detection of subtelomeric chromosomal rearrangements in idiopathic mental retardation. Nat Genet 1995;9:132-140.

6. Knight SJ, Lese CM, Precht KS, Kuc J, Ning Y, Lucas S et al. An optimized set of human telomere clones for studying telomere integrity and architecture. Am J Hum Genet 2000;67:320-332.

7. Kallioniemi A, Kallioniemi OP, Sudar D, Rutovitz D, Gray JW, Waldman F et al. Comparative genomic hybridization for molecular cytogenetic analysis of solid tumors. Science 1992;258:818-821.

8. Solinas-Toldo S, Lampel S, Stilgenbauer S, Nickolenko J, Benner A, Dohner H et al. Matrix-based comparative genomic hybridization: biochips to screen for genomic imbalances. Genes Chromosomes Cancer 1997;20:399-407.

9. Snijders AM, Nowak N, Segraves R, Blackwood S, Brown N, Conroy J et al. Assembly of microarrays for genome-wide measurement of DNA copy number. Nat Genet 2001;29:263-264.

10. Bruder CE, Hirvela C, Tapia-Paez I, Fransson I, Segraves R, Hamilton G et al. High resolution deletion analysis of constitutional DNA from neurofibromatosis type 2 (NF2) patients using microarray-CGH. Hum Mol Genet 2001;10:271-282.

11. Mantripragada KK, Buckley PG, Jarbo C, Menzel U, Dumanski JP. Development of NF2 gene specific, strictly sequence defined diagnostic microarray for deletion detection. J Mol Med 2003;81:443-451.

12. Veltman JA, Jonkers Y, Nuijten I, Janssen I, van d V, Huys E et al. Definition of a critical region on chromosome 18 for congenital aural atresia by arrayCGH. Am J Hum Genet 2003;72:1578-1584.

13. Veltman JA, Schoenmakers EF, Eussen BH, Janssen I, Merkx G, van Cleef B et al. High-throughput analysis of subtelomeric chromosome rearrangements by use of array-based comparative genomic hybridization. Am J Hum Genet 2002;70:12691276.

14. Locke DP, Segraves R, Nicholls RD, Schwartz S, Pinkel D, Albertson DG et al. BAC microarray analysis of 15q11-q13 rearrangements and the impact of segmental duplications. J Med Genet 2004;41:175-182.

15. Buckley PG, Mantripragada KK, Benetkiewicz M, Tapia-Paez I, Diaz DS, Rosenquist $\mathrm{M}$ et al. A full-coverage, high-resolution human chromosome 22 genomic microarray for clinical and research applications. Hum Mol Genet 2002;11:3221-3229.

16. Veltman JA, Yntema HG, Lugtenberg D, Arts H, Briault S, Huys EH et al. High resolution profiling of $\mathrm{X}$ chromosomal aberrations by array comparative genomic hybridisation. J Med Genet 2004;41:425-432.

17. Vissers LE, de Vries BB, Osoegawa K, Janssen IM, Feuth T, Choy CO et al. ArrayBased Comparative Genomic Hybridization for the Genomewide Detection of Submicroscopic Chromosomal Abnormalities. Am J Hum Genet 2003;73:1261-1270.

18. Shaw-Smith C, Redon R, Rickman L, Rio M, Willatt L, Fiegler H et al. Microarray based comparative genomic hybridisation (array-CGH) detects submicroscopic chromosomal deletions and duplications in patients with learning disability/mental retardation and dysmorphic features. J Med Genet 2004;41:241-248.

19. Ishkanian AS, Malloff CA, Watson SK, DeLeeuw RJ, Chi B, Coe BP et al. A tiling resolution DNA microarray with complete coverage of the human genome. Nat Genet 2004;36:299-303.

20. Vissers LE, van Ravenswaaij CM, Admiraal R, Hurst JA, de Vries BB, Janssen IM et al. Mutations in a new member of the chromodomain gene family cause CHARGE syndrome. Nat Genet 2004;36:955-957.
21. Cai WW, Mao JH, Chow CW, Damani S, Balmain A, Bradley A. Genome-wide detection of chromosomal imbalances in tumors using BAC microarrays. Nat Biotechnol 2002;20:393-396.

22. Yu W, Ballif BC, Kashork CD, Heilstedt HA, Howard LA, Cai WW et al. Development of a comparative genomic hybridization microarray and demonstration of its utility with 25 well-characterized 1p36 deletions. Hum Mol Genet 2003;12:21452152 .

23. Shaw CJ, Shaw CA, Yu W, Stankiewicz P, White LD, Beaudet AL et al. Comparative genomic hybridisation using a proximal 17p BAC/PAC array detects rearrangements responsible for four genomic disorders. J Med Genet 2004;41:113-119.

24. Ning Y, Roschke A, Smith ACM, Macha M, Precht K, Riethman H et al. A complete set of human telomeric probes and their clinical application. National Institutes of Health and Institute of Molecular Medicine collaboration. Nat Genet 1996;14:8689.

25. Flint J, Knight S. The use of telomere probes to investigate submicroscopic rearrangements associated with mental retardation. Curr Opin Genet Dev 2003;13:10 16.

26. Katoh M, Shaw C, Xu Q, Van Driessche N, Morio T, Kuwayama H et al. An orderly retreat: Dedifferentiation is a regulated process. Proc Natl Acad Sci USA 2004;101: 7005-7010.

27. Bolstad BM, Irizarry RA, Astrand M, Speed TP. A comparison of normalization methods for high density oligonucleotide array data based on variance and bias. Bioinformatics 2003;19:185-193.

28. Yang YH. Normalization for cDNA microarray data: A robust composite method addressing single and multiple slide systematic variation. Nucleic Acids Res 2002;30: e15.

29. Trask BJ. Fluorescence in situ hybridization: Applications in cytogenetics and gene mapping. Trends Genet 1991;7:149-154.

30. Anderlid BM, Schoumans J, Anneren G, Tapia-Paez I, Dumanski J, Blennow E et al. FISH-mapping of a 100-kb terminal 22q13 deletion. Hum Genet 2002;110:439-443.

31. Guo WJ, Callif-Daley F, Zapata MC, Miller ME. Clinical and cytogenetic findings in seven cases of inverted duplication of $8 p$ with evidence of a telomeric deletion using fluorescence in situ hybridization. Am J Med Genet 1995;58:230-236.

32. Giglio S, Broman KW, Matsumoto N, Calvari V, Gimelli G, Neumann T et al. Olfactory receptor-gene clusters, genomic-inversion polymorphisms, and common chromosome rearrangements. Am J Hum Genet 2001;68:874-883.

33. Riethman H, Ambrosini A, Castaneda C, Finklestein J, Hu XL, Mudunuri U et al. Mapping and initial analysis of human subtelomeric sequence assemblies. Genome Res 2004;14:18-28.

34. Amos-Landgraf JM, Ji Y, Gottlieb W, Depinet T, Wandstrat AE, Cassidy SB et al. Chromosome breakage in the Prader-Willi and Angelman syndromes involves recombination between large, transcribed repeats at proximal and distal breakpoints. Am J Hum Genet 1999;65:370-386.

35. Flint J, Craddock CF, Villegas A, Bentley DP, Williams HJ, Galanello R et al. Healing of broken human chromosomes by the addition of telomeric repeats. Am J Hum Genet 1994;55:505-512.

36. Kostiner DR, Nguyen H, Cox VA, Cotter PD. Stabilization of a terminal inversion duplication of 8 p by telomere capture from 18q. Cytogenet Genome Res 2002;98:912.

37. Katz SG, Schneider SS, Bartuski A, Trask BJ, Massa H, Overhauser J et al. An 18qsyndrome breakpoint resides between the duplicated serpins SCCA1 and SCCA2 and arises via a cryptic rearrangement with satellite III DNA. Hum Mol Genet 1999; 8:87-92.

38. Varley H, Di S, Scherer SW, Royle NJ. Characterization of terminal deletions at 7q32 and 22q13.3 healed by De novo telomere addition. Am J Hum Genet 2000;67:610622.

39. Luciani JJ, de Mas P, Depetris D, Mignon-Ravix C, Bottani A, Prieur M et al. Telomeric $22 \mathrm{q} 13$ deletions resulting from rings, simple deletions, and translocations: cytogenetic, molecular, and clinical analyses of 32 new observations. J Med Genet 2003;40:690-696.

40. Sebat J, Lakshmi B, Troge J, Alexander J, Young J, Lundin P et al. Large-scale copy number polymorphism in the human genome. Science 2004;305:525-528.

41. Fredman D, White SJ, Potter S, Eichler EE, den Dunnen JT, Brookes AJ. Complex SNP-related sequence variation in segmental genome duplications. Nat Genet 2004; 36:861-866.

42. Iafrate AJ, Feuk L, Rivera MN, Listewnik ML, Donahoe PK, Qi Y et al. Detection of large-scale variation in the human genome. Nat Genet 2004;36:949-951.

43. Carter NP. As normal as normal can be? Nat Genet 2004;36:931-932.

44. Carter NP, Vetrie D. Applications of genomic microarrays to explore human chromosome structure and function. Hum Mol Genet 2004;13 Spec No 2:R297R302.

45. Lucito R, Healy J, Alexander J, Reiner A, Esposito D, Chi M et al. Representational oligonucleotide microarray analysis: a high-resolution method to detect genome copy number variation. Genome Res 2003;13:2291-2305. 